\title{
Chapter 35 \\ Self-Assembled Laser-Activated Plasmonic Substrates for High-Throughput, High-Efficiency Intracellular Delivery
}

\author{
Marinna Madrid, Nabiha Saklayen, Marinus Huber, Nicolas Vogel, and \\ Eric Mazur
}

\begin{abstract}
Delivering material into cells is important for a diverse range of biological applications, including gene therapy, cellular engineering and imaging. We presented a plasmonic substrate for delivering membrane-impermeable material into cells at high throughput and high efficiency while maintaining cell viability. The substrate fabrication is based on an affordable and fast colloidal self-assembly process. When illuminated with a femtosecond laser, the light interacts with the electrons at the surface of the metal substrate, creating localized surface plasmons that form bubbles via energy dissipation in the surrounding medium. These bubbles come into close contact with the cell membrane to form transient pores and enable entry of membrane-impermeable material via diffusion. We performed proof of principle experiments using both a femtosecond laser and a nanosecond laser system. We used fluorescence microscopy to verify delivery of membraneimpermeable dye into HeLa CCL-2 cells and to verify cell viability after laser treatment. Our findings indicate that self-assembled plasmonic substrates may be an affordable, flexible tool for high-throughput, high-efficiency delivery of material into mammalian cells.
\end{abstract}

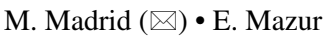 \\ School of Engineering and Applied Sciences, Harvard University, Cambridge, MA, USA \\ e-mail: marinnamadrid@gmail.com; mazur@physics.harvard.edu \\ N. Saklayen \\ Physics Department, Harvard University, Cambridge, MA, USA \\ e-mail: nsaklayen@gmail.com \\ M. Huber \\ Max Planck Institute, Munich, Germany \\ N. Vogel \\ University of Erlangen, Erlangen, Germany

